\title{
ORDERED DSmT AND APPLICATION TO THE DEFINITION OF CONTINUOUS DSm MODELS
}

\author{
Frédéric DAMBREVILLE
}

\begin{abstract}
When implementing the DSmT, a difficulty may arise from the possible huge dimension of hyperpower sets, which are indeed free structures. However, it is possible to reduce the dimension of these structures by involving logical constraints. In this paper, the logical constraints will be related to a predefined order over the logical propositions. The use of such orders and their resulting logical constraints will ensure a great reduction of the model complexity. Such results will be applied to the definition of continuous DSm models. In particular, a simplified description of the continuous impreciseness is considered, based on impreciseness intervals of the sensors. From this viewpoint, it is possible to manage the contradictions between continuous sensors in a DSmT manner, while the complexity of the model stays handlable.
\end{abstract}

Keywords: Evidence Theory, Continuous DSmT, Probability, Boolean Algebra, Hyperpower set

\section{Introduction}

Recent advances[1] in the Dezert Smarandache Theory have shown that this theory was able to handle the contradiction between propositions in a quite flexible way. This new theory has been already applied in different domains; eg.:

- Data asociation in target tracking [2],

- Environmental prediction [3].

Although free DSm models are defined over hyperpower sets, which sizes evolve exponentially with the number of atomic propositions, it appears that the manipulation of the fusion rule is still manageable for practical problems reasonnably well shaped. Moreover, the hybrid DSm models are of lesser complexity.

If DSmT works well for discret spaces, the manipulation of continous DSm models is still an unknown. A question first arises: what could be an hyperpower set for a continuous DSm model? Such first issue does not arises so dramatically in Dempster Shafer 
Theory or for Transfer Belief Models[4]. In DST, a continuous proposition could just be a measurable subset. On the other hand, a free DSm model, defined over an hyperpower set, will imply that any pair of propositions will have a non empty intersection. This is desappointing, since the notion of point (a minimal non empty proposition) does not exist anymore in an hyperpower set.

But even if it is possible to define a continuous propositional model, the manipulation of continuous basic belief assignment is still an issue[5][6]. In [5], Ristic and Smets proposed a restriction of the bba to intervals of $\mathbb{R}$. It was then possible to derive a mathematical relation between a continuous bba density and its Bel function.

In this paper, the construction of continuous DSm models is proposed. This construction is based on a constrained model, where the logical contraints are implied by the definition of an order relation over the propositions.

A one-dimension DSm model will be implemented, where the definition of the basic belief assignment relies on a generalized notion of intervals. Although this construction has been fulfilled on a different ground, it shares some surprizing similarities with Ristic and Smets viewpoint. As in [5], the bba will be seen as density defined over a 2-dimension measurable space. We will be able to derive the Belief function from the basic belief assignment, by applying an integral computation. At last, the conjunctive fusion operator, $\oplus$, is derived by a rather simple integral computation.

Section 2 makes a quick introduction of the Dezert Smarandache Theory. Section 3 is about ordered DSm models. In section 4, a continuous DSm model is defined. This method is restricted to only one dimension. The related computation methods are detailed. In section 5, our algorithmic implementation is described and an example of computation is given. The paper is then concluded.

\section{A short introduction to the DSmT}

\subsection{Basis}

The theory and its meaning are widely explained in [1].

The Dezert Smarandache Theory belongs to the family of Evidence Theories. As the Dempster Shafer Theory[7][8] or the Transferable Belief Models[4], the DSmT is a framework for fusing belief informations, originating from independent sensors. Free DSm models are defined over Hyperpower sets, which are fully open-world extensions of sets. It is possible to restrict this full open-world hypothesis by adding propositional constraints, resulting in the definition of an hybrid Dezert Smarandache model.

Hyperpower set. Let $\Phi=\left\{\phi_{i} / i \in I\right\}$ be a set of propositions (finite or infinite). The hyperpower set $\langle\Phi\rangle$ is the boolean pre-algebra freely generated by $\Phi$ and the 
boolean operators $\wedge(A N D)$ and $\vee(O R)$. It does not contains the negation $\neg$.

Example.

$$
\begin{aligned}
<a, b, c>=\{ & a, b, c, a \wedge b \wedge c, a \vee b \vee c, a \wedge b, b \wedge c, c \wedge a \\
& a \vee b, b \vee c, c \vee a,(a \wedge b) \vee c,(b \wedge c) \vee a,(c \wedge a) \vee b, \\
& (a \vee b) \wedge c,(b \vee c) \wedge a,(c \vee a) \wedge b,(a \wedge b) \vee(b \wedge c) \vee(c \wedge a)\}
\end{aligned}
$$

It is easy to verify that this set is left unchanged by any application of the operators $\wedge$ and $\vee$. For example:

$$
(a \wedge b) \wedge((b \wedge c) \vee a)=(a \wedge b \wedge b \wedge c) \vee(a \wedge b \wedge a)=a \wedge b
$$

Definition. The relation $\subset$ is defined over $<\Phi>$ by:

$$
\forall \phi, \psi \in<\Phi>, \quad \phi \subset \psi \stackrel{\Delta}{\Longleftrightarrow} \phi \wedge \psi=\phi
$$

Dezert Smarandache Model. Assume that $\Phi$ is a finite set. A Dezert Smarandache model (DSmm) is a pair $(\Phi, m)$, where $\Phi$ is a set of propositions and the basic belief assignment $m$ is a non negatively valued function defined over $<\Phi>$ such that:

$$
\sum_{\phi \in<\Phi>} m(\phi)=1
$$

Belief Function. Assume that $\Phi$ is a finite set. The belief function Bel related to a bba $m$ is defined by:

$$
\forall \phi \in<\Phi>, \operatorname{Bel}(\phi)=\sum_{\psi \in<\Phi>: \psi \subset \phi} m(\psi)
$$

The equation (1) is invertible:

$$
\forall \phi \in<\Phi>, m(\phi)=\operatorname{Bel}(\phi)-\sum_{\psi \in<\Phi>: \psi \subsetneq \phi} m(\psi) .
$$

Fusion rule. Assume that $\Phi$ is a finite set. For a given universe $\Phi$, and two basic belief assignments $m_{1}$ and $m_{2}$, associated to independent sensors, the fused basic belief assignment is $m_{1} \oplus m_{2}$, defined by:

$$
m_{1} \oplus m_{2}(\phi)=\sum_{\psi_{1}, \psi_{2} \in<\Phi>: \psi_{1} \wedge \psi_{2}=\phi} m_{1}\left(\psi_{1}\right) m_{2}\left(\psi_{2}\right) .
$$




\subsection{Some extensions}

Between sets and hyperpower sets. Sets and hyperpower sets are tightly related structures. First at all, a set (with $\cap, \cup$ operators and complement) is a boolean algebra, while an hyperpower set is a free boolean pre-algebra. It is clear that a free boolean pre-algebra could be completed to a boolean algebra, so that an hyperpower set could be seen as a substructure of a set. More precisely $\langle\Phi>\subset \mathcal{B}(\Phi)$ where $\mathcal{B}(\Phi)$ is the free boolean algebra generated by $\Phi$. In particular, when $\Phi$ is finite, the boolean algebra $\mathcal{B}(\Phi)$ is generated by:

$$
\left\{\bigwedge_{i \in I} \epsilon_{i} / \forall i \in I, \epsilon_{i} \in\left\{\phi_{i}, \neg \phi_{i}\right\}\right\} .
$$

Followingly, $\mathcal{B}(\Phi)$ is isomorph to a set structure of $2^{\operatorname{card}(I)}$ elements, in the finite case. Conversely, a set could be interpreted as a constrained pre-algebra, ie. a "constrained hyperpower set". More precisely, when $\Phi$ is a finite set, ${ }^{1}$ this set is isomorph to the boolean pre-algebra generated by $\Phi, \wedge$ and $\vee$, and verifying the logical constraints:

$$
\forall i, j \in I, i \neq j \Rightarrow \phi_{i} \wedge \phi_{j}=\perp \text {. }
$$

In this construction, the empty proposition $\perp$ has been implicitely defined. However, it is possible (see later) to build constrained pre-algebra without the adjunction of the empty proposition $\perp$.

One advantage of constrained boolean pre-algebra is that they are less complex and "fractalized" than a simple hyperpower set. Jean Dezert and Florentin Smarandache have extended the DSmT fusion operator so has to involve any kind of pre-algebra (hybrid DSmT [1]). In this presentation, we will only focuse on pre-algebra constrained without adjunction of $\perp$, and in this case, the fusion operator of the free DSmT is kept unchanged.

Partially open world without $\perp$. Let $\Gamma \subset<\Phi>\times\langle\Phi>$ and define the prealgebra $\left\langle\Phi>_{\Gamma}\right.$ generated by $\Phi, \wedge, \vee$ and constrained by:

$$
\forall(\phi, \psi) \in \Gamma, \phi=\psi \text {. }
$$

Example. Let consider again the case $\Phi=\{a, b, c\}$. But now, let us introduce the constraints $a \wedge b=a \wedge c=b \wedge c$, which means (for example) using a set $\Gamma=$ $\{(a \wedge b, a \wedge c),(a \wedge c, b \wedge c)\}$. Then it is deduced $a \wedge b=a \wedge c=b \wedge c=a \wedge b \wedge c$. It follows $(a \wedge b) \vee c=c,(b \wedge c) \vee a=a$ and $(c \wedge a) \vee b=b$. It is also deduced $(a \vee b) \wedge c=(b \vee c) \wedge a=(c \vee a) \wedge b=(a \wedge b) \vee(b \wedge c) \vee(c \wedge a)=a \wedge b \wedge c$. By discarding these cases from the free hyperpower $\langle a, b, c\rangle$, it is deduced:

$$
<a, b, c>_{\Gamma}=\{a, b, c, a \wedge b \wedge c, a \vee b \vee c, a \vee b, b \vee c, c \vee a\}
$$

${ }^{1}$ When $\Phi$ is infinite, this result requires "infinite $\vee$-ing." 
It is noticed that $\left\langle\Phi>_{\Gamma}\right.$ is left unchanged by any application of the operators $\wedge$ and $\vee$ (and does not contains the external proposition $\perp$ ). When $\Phi$ is finite, the definition of bba $m$, belief Bel and fusion $\oplus$ is thus kept unchanged.

- A basic belief assignment $m$ is a non negatively valued function defined over $<\Phi>_{\Gamma}$ such that:

$$
\sum_{\phi \in<\Phi>_{\Gamma}} m(\phi)=1
$$

- The belief function Bel related to a bba $m$ is defined by:

$$
\forall \phi \in<\Phi>_{\Gamma}, \operatorname{Bel}(\phi)=\sum_{\psi \in<\Phi>_{\Gamma}: \psi \subset \phi} m(\psi) .
$$

- Being given two basic belief assignments $m_{1}$ and $m_{2}$, the fused basic belief assignment $m_{1} \oplus m_{2}$ is defined by:

$$
m_{1} \oplus m_{2}(\phi)=\sum_{\psi_{1}, \psi_{2} \in<\Phi>_{\Gamma}: \psi_{1} \wedge \psi_{2}=\phi} m_{1}\left(\psi_{1}\right) m_{2}\left(\psi_{2}\right) .
$$

These extended definitions will be applied subsequently.

\section{Ordered DSm model}

In order to reduce the complexity of the free DSm model, it is necessary to introduce logical constraints which will lower the size of the pre-algebra. Such constraints may appear clearly in the hypotheses of the problem. In this case, constraints come naturally and approximations may not be required. However, when the model is too complex and there are no explicit constraints for reducing this complexity, it is necessary to approximate the model by introducing some new constraints. Two rules should be applied then:

- Only weaken informations ${ }^{2}$; do not produce information from nothing,

- minimize the information weakening.

First point garantees that the approximation does not introduce false information. But some significant informations (eg. contradictions) are possibly missed. This drawback should be avoided by second point.

In order to build a good approximation policy, some external knowledge, like distance or order relation among the propositions could be used. Behind these relations will be assumed some kind of distance between the informations: more are the informations distant, more are their conjunctive combination valuable.

\footnotetext{
${ }^{2}$ Typically, a constraint like $\phi \wedge \psi \wedge \eta=\phi \wedge \psi$ will weaken the information, by erasing $\eta$ from $\phi \wedge \psi \wedge \eta$.
} 


\subsection{Ordered atomic propositions}

Let $(\Phi, \leq)$ be an ordered set of propositions. This order relation is assumed to describe the relative distance between the information. For example, the relation $\phi \leq \psi \leq \eta$ implies that $\phi$ and $\psi$ are closer informations than $\phi$ and $\eta$. Thus, the information contained in $\phi \wedge \eta$ is stronger than the information contained in $\phi \wedge \psi$. Of course, this comparison does not matter when all the information is kept, but when approximations are necessary, it will be useful to be able to choose the best information.

Sketchy example. Assume that 3 independent sensors are giving 3 measures about a continuous parameter, that is $x, y$ and $z$. The parameters $x, y, z$ are assumed to be real values, not of the set $\mathbb{R}$ but of its "pre-algebraic" extension (theoretical issues will be clarified later $\left.{ }^{3}\right)$. The fused information could be formalized by the proposition $x \wedge y \wedge z$ (in a DSmT viewpoint). What happen if we want to reduce the information by removing a proposition. Do we keep $x \wedge y, y \wedge z$ or $x \wedge z$ ? This is of course an information weakening. But it is possible that one information is better than an other. At this stage, the order between the values $x, y, z$ will be involved. Assume for example that $x \leq y<z$. It is clear that the proposition $x \wedge z$ indicates a greater contradiction than $x \wedge y$ or $y \wedge z$. Thus, the proposition $x \wedge z$ is the one which should be kept! The discarding constraint $x \leq y \leq z \Rightarrow x \wedge y \wedge z=x \wedge z$ is implied then.

\subsection{Associated pre-algebra and complexity.}

In regard to the previous example, the pre-algebra associated to the ordered propositions $(\Phi, \leq)$ is $\left\langle\Phi>_{\Gamma}\right.$, where $\Gamma$ is defined by:

$$
\Gamma=\{(\phi \wedge \psi \wedge \eta, \phi \wedge \eta) / \phi, \psi, \eta \in \Phi \text { and } \phi \leq \psi \leq \eta\}
$$

The following property give an approximative bound of the size of $\langle\Phi\rangle_{\Gamma}$ in the case of a total order.

Proposition 1 Assume that $(\Phi, \leq)$ is totally ordered. Then, $\langle\Phi\rangle_{\Gamma}$ is a substructure of the set $\Phi^{2}$.

proof. Since the order is total, first notice that the added constraints are:

$$
\forall \phi, \psi, \eta \in \Phi, \phi \wedge \psi \wedge \eta=\min \{\phi, \psi, \eta\} \wedge \max \{\phi, \psi, \eta\}
$$

Now, for any $\phi \in \Phi$, define $\breve{\phi}$ by:

$$
\underline{\breve{\phi}}=\left\{\left(\varphi_{1}, \varphi_{2}\right) \in \Phi^{2} / \varphi_{1} \leq \phi \leq \varphi_{2}\right\}
$$

\footnotetext{
${ }^{3}$ In particular, as we are working in a pre-algebra, $x \wedge y$ makes sense and it is possible that $x \wedge y \neq \perp$ even when $x \neq y$
} 
It is noteworthy that:

$$
\breve{\phi} \cap \breve{\psi}=\left\{\left(\varphi_{1}, \varphi_{2}\right) \in \Phi^{2} / \varphi_{1} \leq \min \{\phi, \psi\} \text { and } \max \{\phi, \psi\} \leq \varphi_{2}\right\}
$$

and

$\breve{\phi} \cap \breve{\psi} \cap \breve{\eta}=\left\{\left(\varphi_{1}, \varphi_{2}\right) \in \Phi^{2} / \varphi_{1} \leq \min \{\phi, \psi, \eta\}\right.$ and $\left.\max \{\phi, \psi, \eta\} \leq \varphi_{2}\right\}$.

By defining $m=\min \{\phi, \psi, \eta\}$ and $M=\max \{\phi, \psi, \eta\}$, it is deduced:

$$
\breve{\phi} \cap \breve{\psi} \cap \breve{\eta}=\breve{m} \cap \breve{M} \text {. }
$$

Let $\mathcal{A} \subset \mathcal{P}\left(\Phi^{2}\right)$ be generated by $\left.\breve{\phi}\right|_{\phi \in \Phi}$ with $\cap$ and $\cup$, ie.

$$
\mathcal{A}=\bigcup_{n \geq 0}\left\{\bigcup_{k=1}^{n}\left(\breve{\phi}_{k} \cap \breve{\psi}_{k}\right) / \forall k, \breve{\phi}_{k}, \breve{\psi}_{k} \in \Phi\right\} .
$$

Then, by (3) is defined the mapping:

$$
\smile:\left\{\begin{array}{l}
<\Phi>_{\Gamma}^{n} \longrightarrow \mathcal{A} \\
\bigvee_{k=1}^{n} \bigwedge_{l=1}^{n_{k}} \phi_{k, l} \longmapsto \bigcup_{k=1}^{n} \bigcap_{l=1}^{n_{k}} \breve{\phi}_{k, l} \quad, \quad \text { where } \phi_{k, l} \in \Phi
\end{array}\right.
$$

which is an onto morphism of pre-algebra.

Lemma 1 Assume:

$$
\bigcup_{k=1}^{n}\left(\breve{\phi}_{k}^{1} \cap \breve{\phi}_{k}^{2}\right) \subset \bigcup_{l=1}^{m}\left(\breve{\psi}_{l}^{1} \cap \breve{\psi}_{l}^{2}\right) \quad, \quad \text { where } \phi_{k}^{j}, \psi_{l}^{j} \in \Phi .
$$

Then:

$$
\forall k, \exists l, \min \left\{\phi_{k}^{1}, \phi_{k}^{2}\right\} \leq \min \left\{\psi_{l}^{1}, \psi_{l}^{2}\right\} \text { and } \max \left\{\phi_{k}^{1}, \phi_{k}^{2}\right\} \geq \max \left\{\psi_{l}^{1}, \psi_{l}^{2}\right\}
$$

and

$$
\forall k, \exists l, \breve{\phi}_{k}^{1} \cap \breve{\phi}_{k}^{2} \subset \breve{\psi}_{l}^{1} \cap \breve{\psi}_{l}^{2}
$$

Proof of lemma. Let $k \in \llbracket 1, n \rrbracket$.

Define $m=\min \left\{\phi_{k}^{1}, \phi_{k}^{2}\right\}$ and $M=\max \left\{\phi_{k}^{1}, \phi_{k}^{2}\right\}$.

Then holds $(m, M) \in \breve{\phi}_{k}^{1} \cap \breve{\phi}_{k}^{2}$, implying $(m, M) \in \bigcup_{l=1}^{m}\left(\breve{\psi}_{l}^{1} \cap \breve{\psi}_{l}^{2}\right)$.

Let $l$ be such that $(m, M) \in \breve{\psi}_{l}^{1} \cap \breve{\psi}_{l}^{2}$.

Then $m \leq \min \left\{\psi_{l}^{1}, \psi_{l}^{2}\right\}$ and $M \geq \max \left\{\psi_{l}^{1}, \psi_{l}^{2}\right\}$.

Followingly, $\breve{\phi}_{k}^{1} \cap \breve{\phi}_{k}^{2} \subset \breve{\psi}_{l}^{1} \cap \breve{\psi}_{l}^{2}$. 
From $\min \left\{\phi_{k}^{1}, \phi_{k}^{2}\right\} \leq \min \left\{\psi_{l}^{1}, \psi_{l}^{2}\right\}$ and $\max \left\{\phi_{k}^{1}, \phi_{k}^{2}\right\} \geq \max \left\{\psi_{l}^{1}, \psi_{l}^{2}\right\}$ is also deduced $\left(\phi_{k}^{1} \wedge \phi_{k}^{2}\right) \wedge\left(\psi_{l}^{1} \wedge \psi_{l}^{2}\right)=\phi_{k}^{1} \wedge \phi_{k}^{2}$ (definition of $\Gamma$ ).

This property just means $\phi_{k}^{1} \wedge \phi_{k}^{2} \subset \psi_{l}^{1} \wedge \psi_{l}^{2}$. It is lastly deduced:

Lemma 2 Assume:

$$
\bigcup_{k=1}^{n}\left(\breve{\phi}_{k}^{1} \cap \breve{\phi}_{k}^{2}\right) \subset \bigcup_{l=1}^{m}\left(\breve{\psi}_{l}^{1} \cap \breve{\psi}_{l}^{2}\right) \quad, \quad \text { where } \phi_{k}^{j}, \psi_{l}^{j} \in \Phi .
$$

Then:

$$
\bigvee_{k=1}^{n}\left(\phi_{k}^{1} \wedge \phi_{k}^{2}\right) \subset \bigvee_{l=1}^{m}\left(\psi_{l}^{1} \wedge \psi_{l}^{2}\right)
$$

From this lemma, it is deduced that $\smile$ is one to one.

At last $\smile$ is an isomorphism of pre-algebra, and $\left\langle\Phi>_{\Gamma}\right.$ is a substructure of $\Phi^{2}$.

\subsection{General properties of the model}

In the next section, the previous construction will be extended to the continuous case, ie. $(\mathbb{R}, \leq)$. However, a strict logical manipulation of the propositions is not sufficient and instead a measurable generalization of the model will be used. It has been seen that a proposition of $\left\langle\Phi>_{\Gamma}\right.$ could be described as a subset of $\Phi^{2}$. In this subsection, the proposition model will be characterized precisely. This characterization will be used and extended in the next section to the continuous case.

Proposition 2 Let $\phi \in<\Phi>_{\Gamma}$.

Then $\smile(\phi) \subset \mathcal{T}$, where $\mathcal{T}=\left\{(\phi, \psi) \in \Phi^{2} / \phi \leq \psi\right\}$.

Proof. Obvious, since $\forall \phi \in \Phi, \breve{\phi} \subset \mathcal{T}$.

Definition 1 A subset $\theta \subset \Phi^{2}$ is increasing if and only if:

$$
\forall(\phi, \psi) \in \theta, \forall \eta \leq \phi, \forall \zeta \geq \psi,(\eta, \zeta) \in \theta
$$

Let $\mathcal{U}=\{\theta \subset \mathcal{T} / \theta$ is increasing $\}$ be the set of increasing subsets of $\mathcal{T}$. Notice that the intersection or the union of increasing subsets are increasing subsets, so that $(\mathcal{U}, \cap, \cup)$ is a pre-algebra. 
Proposition 3 For any choice of $\Phi,\left\{\smile(\phi) / \phi \in<\Phi>_{\Gamma}\right\} \subset \mathcal{U}$. When $\Phi$ is finite, $\mathcal{U}=\left\{\smile(\phi) / \phi \in<\Phi>_{\Gamma}\right\}$.

Proof of $\supset$. Obvious, since $\breve{\phi}$ is inceasing for any $\phi \in \Phi$.

Proof of $\subset$. Let $\theta \in \mathcal{U}$ and let $(a, b) \in \theta$.

Since $\breve{a} \cap \breve{b}=\left\{(\alpha, \beta) \in \Phi^{2} / \alpha \leq a\right.$ and $\left.\beta \geq b\right\}$ and $\theta$ is increasing, it follows $\breve{a} \cap \breve{b} \subset \theta$.

At last, $\theta=\bigcup_{(a, b) \in \theta} \breve{a} \cap \breve{b}=\smile\left(\bigvee_{(a, b) \in \theta} a \wedge b\right)$.

Notice that $\bigvee_{(a, b) \in \theta} a \wedge b$ is actually defined, since $\theta$ is finite when $\Phi$ is finite.

When infinite $V$-ing are allowed, notice that $\mathcal{U}$ may be considered as a model for $<\Phi>_{\Gamma}$ even if $\Phi$ is infinite. In the next section, the continuous pre-algebra related to $(\mathbb{R}, \leq)$ will be modelled by the measurable increasing subsets of $\left\{(x, y) \in \mathbb{R}^{2} / x \leq y\right\}$.

\section{Continuous DSm model}

In this section, the case $\Phi=\mathbb{R}$ is considered.

Typically, in a continuous model, it will be necessary to manipulate any measurable proposition, and for example intervals. It comes out that most intervals could not be obtained by a finite logical combinaison of the atomic propositions, but rather by infinite combinations. For example, considering the set formalism, it is obtained $[a, b]=$ $\bigcup_{x \in[a, b]}\{x\}$, which suggests the definition of the infinite disjunction " $\bigvee_{x \in[a, b]} x$ ". It is known that infinite disjunctions are difficult to handle in a logic. It is better to manipulate the models directly. The prealgebra to be constructed should verify the property $x \leq y \leq z \Rightarrow x \wedge y \wedge z=x \wedge z$. As discussed previously and since infinitary disjunctions are allowed, a model for such algebra are the measurable increasing subsets.

\subsection{Measurable increasing subsets}

A measurable subset $A \subset \mathbb{R}^{2}$ is a measurable increasing subset if:

$$
\left\{\begin{array}{l}
\forall(x, y) \in A, x \leq y \\
\forall(x, y) \in A, \forall a \leq x, \forall b \geq y,(a, b) \in A
\end{array}\right.
$$

The set of measurable increasing subsets is denoted $\mathcal{U}$.

Example. Let $f: \mathbb{R} \rightarrow \mathbb{R}$ be a non decreasing measurable mapping such that $f(x) \geq x$ for any $x \in \mathbb{R}$. The set $\left\{(x, y) \in \mathbb{R}^{2} / f(x) \leq y\right\}$ is a measurable increasing subset. 
“Points". For any $x \in \mathbb{R}$, the measurable increasing subset $\breve{x}$ is defined by:

$$
\breve{x}=\left\{(a, b) \in \mathbb{R}^{2} / a \leq x \leq b\right\} .
$$

The set $\breve{x}$ is of course a model for the point $x \in \mathbb{R}$ within the pre-algebra (refer to section 3).

Generalized intervals. A particular class of increasing subsets, the generalized intervals, will be useful in the sequel.

For any $x \in \mathbb{R}$, the measurable sets $\grave{x}$ and $x$ are then defined by:

$$
\left\{\begin{array}{l}
\grave{x}=\left\{(a, b) \in \mathbb{R}^{2} / a \leq b \text { and } x \leq b\right\}, \\
\dot{x}=\left\{(a, b) \in \mathbb{R}^{2} / a \leq b \text { and } a \leq x\right\} .
\end{array}\right.
$$

The following properties are derived:

$$
\breve{x}=\grave{x} \cap \grave{x}, \grave{x}=\bigcup_{z \in[x,+\infty[} \breve{z} \quad \text { and } \quad \dot{x}=\bigcup_{z \in]-\infty, x]} \breve{z}
$$

Moreover, for any $x, y$ such that $x \leq y$, it comes:

$$
\grave{x} \cap \dot{y}=\bigcup_{z \in[x, y]} \breve{z} .
$$

As a conclusion, the set $\grave{x}, \grave{x}$ and $\grave{x} \cap y$ (with $x \leq y$ ) are the respective models for the intervals $[x,+\infty[]-,\infty, x]$ and $[x, y]$ within the pre-algebra. Naturally, the accents and 'are used respectively for opening and closing the intervals.

At last, the set $\grave{x} \cap \dot{y}$, where $x, y \in \mathbb{R}$ are not constrained, constitutes a generalized definition of the notion of interval. In the case $x \leq y$, it works like "classical" interval, but in the case $x>y$, it is obtained a new class of intervals with negative width. Whatever, $\grave{x} \cap y$ comes with a non empty inner, and may have a non zero measure.

The width $\delta=\frac{y-x}{2}$ of the interval $\grave{x} \cap y$ could be considered as a measure of contradiction associated with this proposition, while its center $\mu=\frac{x+y}{2}$ should be considered as its median value. The interpretation of the measure of contradiction is left to the human. Typically, a possible interpretation could be:

- $\delta<0$ means contradictory informations,

- $\delta=0$ means exact informations,

- $\delta>0$ means imprecise informations. 
It is also noteworthy that the set of generalized intervals

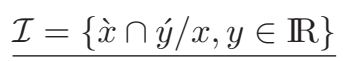

is left unchanged by the operator $\cap$, as seen in the following proposition 4 :

Proposition 4 (Stability) Let $x_{1}, x_{2}, y_{1}, y_{2} \in \mathbb{R}$.

Define $x=\max \left\{x_{1}, x_{2}\right\}$ and $y=\min \left\{y_{1}, y_{2}\right\}$.

Then $\left(\grave{x}_{1} \cap \grave{y}_{1}\right) \cap\left(\grave{x}_{2} \cap \dot{y}_{2}\right)=\grave{x} \cap \dot{y}$.

Proof is obvious.

This last property make possible the definition of basic belief assignment over generalized intervals only. This assumption is clearly necessary in order to reduce the complexity of the evidence modelling. Behind this assumption is the idea that a continuous measure is described by an imprecision/contradiction around the sensored value. Such hypothesis has been made by Smets and Risic[5]. From now on, all the defined bba will be zeroed outside $\mathcal{I}$. Now, since $\mathcal{I}$ is invariant by $\cap$, it is implied that all the bba which will be manipulated, from sensors or after fusion, will be zeroed outside $\mathcal{I}$. This makes the basic belief assignments equivalent to a density over the 2-dimension space $\mathbb{R}^{2}$.

\subsection{Definition and manipulation of the belief}

The definitions of bba, belief and fusion result directly from section 2, but of course the bba becomes density and the summations are replaced by integrations.

Basic Belief Assignment. As discussed previously, it is hypothesized that the measures are characterized by a precision interval around the sensored values. In addition, there is an unknown about the measure which is translated into a basic belief assignment over the precision intervals.

According to this hypotheses, a bba will be a non negatively valued function $m$ defined over $\mathcal{U}$, zeroed outside $\mathcal{I}$ (set of generalized intervals), and such that:

$$
\int_{x, y \in \mathbb{R}} m(\grave{x} \cap \grave{y}) d x d y=1 .
$$

Belief function. The function of belief, Bel, is defined for any measurable proposition $\phi \in \mathcal{U}$ by:

$$
\operatorname{Bel}(\phi)=\int_{\grave{x} \cap y ́} m(\grave{x} \cap \grave{y}) d x d y .
$$

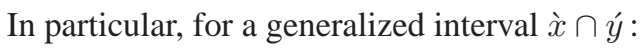

$$
\operatorname{Bel}(\grave{x} \cap \dot{y})=\int_{u=x}^{+\infty} \int_{v=-\infty}^{y} m(\grave{u} \cap \dot{v}) d u d v .
$$


Fusion rule. Being given two basic belief assignments $m_{1}$ and $m_{2}$, the fused basic belief assignment $m_{1} \oplus m_{2}$ is defined by the curviline integral:

$$
m_{1} \oplus m_{2}\left(\grave{x} \cap y^{\prime}\right)=\int_{\mathcal{C}=\{(\phi, \psi) / \phi \cap \psi=\grave{x} \cap \dot{y}\}} m_{1}(\phi) m_{2}(\psi) d \mathcal{C} .
$$

Now, from hypothesis it is assumed that $m_{i}$ is positive only for intervals of the form $\grave{x}_{i} \cap \dot{y}_{i}$. Proposition 4 implies:

$$
\grave{x}_{1} \cap \grave{y}_{1} \cap \grave{x}_{2} \cap \grave{y}_{2}=\grave{x} \cap \grave{y} \text { where }\left\{\begin{array}{l}
x=\max \left\{x_{1}, x_{2}\right\}, \\
y=\min \left\{y_{1}, y_{2}\right\} .
\end{array}\right.
$$

It is then deduced:

$$
\begin{aligned}
& m_{1} \oplus m_{2}\left(\grave{x} \cap y^{\prime}\right)=\int_{-\infty}^{x} \int_{y}^{+\infty} m_{1}(\grave{x} \cap y) m_{2}\left(\grave{x}_{2} \cap \dot{y}_{2}\right) d x_{2} d y_{2} \\
& +\int_{-\infty}^{x} \int_{y}^{+\infty} m_{1}\left(\grave{x}_{1} \cap \grave{y}_{1}\right) m_{2}(\grave{x} \cap \grave{y}) d x_{1} d y_{1} \\
& +\int_{-\infty}^{x} \int_{y}^{+\infty} m_{1}\left(\grave{x}_{1} \cap y^{\prime}\right) m_{2}\left(\grave{x} \cap \dot{y}_{2}\right) d x_{1} d y_{2} \\
& +\int_{-\infty}^{x} \int_{y}^{+\infty} m_{1}\left(\grave{x} \cap \dot{y}_{1}\right) m_{2}\left(\grave{x}_{2} \cap \hat{y}\right) d x_{2} d y_{1} \text {. }
\end{aligned}
$$

In particular, it is now justified that a bba, from sensors or fused, will always be zeroed outside $\mathcal{I}$.

\section{Implementation of the continuous model}

Setting. In this implementation, the study has been restricted to the interval $[-1,1]$ instead of $\mathbb{R}$. The previous results still hold by trunctating over $[-1,1]$. In particular, any bba $m$ is zeroed outside $\mathcal{I}_{-1}^{1}=\{\grave{x} \cap \grave{y} / x, y \in[-1,1]\}$ and its related belief function is defined by:

$$
\operatorname{Bel}\left(\grave{x} \cap y^{\prime}\right)=\int_{u=x}^{1} \int_{v=-1}^{y} m\left(\grave{u} \cap v^{\prime}\right) d u d v
$$


for any generalized interval of $\mathcal{I}_{-1}^{1}$. The bba resulting of the fusion of two bbas $m_{1}$ and $m_{2}$ is defined by:

$$
\begin{aligned}
m_{1} \oplus & m_{2}(\grave{x} \cap \dot{y})=\int_{-1}^{x} \int_{y}^{1} m_{1}(\grave{x} \cap \dot{y}) m_{2}\left(\grave{x}_{2} \cap \dot{y}_{2}\right) d x_{2} d y_{2} \\
& +\int_{-1}^{x} \int_{y}^{1} m_{1}\left(\grave{x}_{1} \cap \dot{y}_{1}\right) m_{2}(\grave{x} \cap \grave{y}) d x_{1} d y_{1} \\
& +\int_{-1}^{x} \int_{y}^{1} m_{1}\left(\grave{x}_{1} \cap \dot{y}\right) m_{2}\left(\grave{x} \cap \dot{y}_{2}\right) d x_{1} d y_{2} \\
& +\int_{-1}^{x} \int_{y}^{1} m_{1}\left(\grave{x} \cap \dot{y}_{1}\right) m_{2}\left(\grave{x}_{2} \cap \dot{y}\right) d x_{2} d y_{1} .
\end{aligned}
$$

Method. A theorical computation of these integrals seems uneasy. An approximation of the densities and of the integrals has been considered. More precisely, the densities have been approximitated by means of 2-dimension Chebyshev polynomials, which have several good properties:

- The approximation grows quickly with the degree of the polynomial, without oscilliation phenomena,

- The Chebyshev transform is quite related to the fourier transform, which makes the parameters of the polynoms really quickly computable by means of a Fast Fourier Transform,

- Integration is easy to compute.

In our tests, we have chosen a Chebyshev approximation of degree $128 \times 128$, which is more than sufficient for an almost exact computation.

Example. Two bba $m_{1}$ and $m_{2}$ have been constructed by normalizing the following functions $m m_{1}$ and $m m_{2}$ defined over $[-1,1]^{2}$ :

$$
m m_{1}(\grave{x} \cap \grave{y})=\exp \left(-(x+1)^{2}-y^{2}\right)
$$

and

$$
m m_{2}\left(\grave{x} \cap y^{\prime}\right)=\exp \left(-x^{2}-(y-1)^{2}\right) .
$$

The fused bba $m_{1} \oplus m_{2}$ and the respective belief function $b_{1}, b_{2}, b_{1} \oplus b_{2}$ have been computed. This computation has been instantaneous. All functions have been represented in the figures 1 to 8 . 
pre-bba $\mathrm{mm}_{1}$

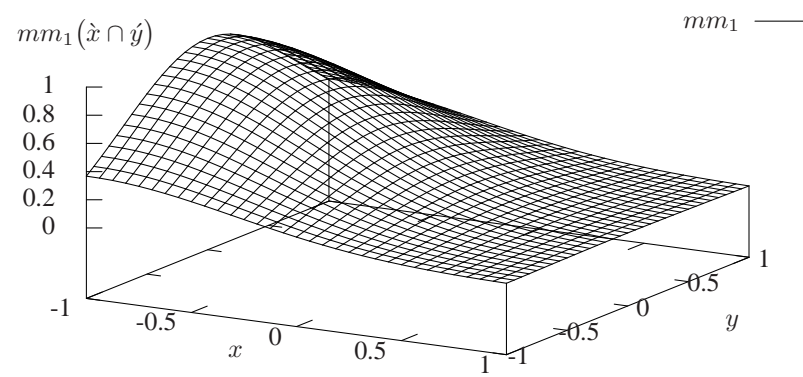

Figure 1: Non normalized bba $m m_{1}$

Interpretation. The bba $m_{1}$ is a density centered around the interval $[-1,0]$, while $m_{2}$ is a density centered around $[0,1]$. This explains why the belief $b_{1}$ increases faster from the interval $[-1,-1]$ to $[-1,1]$ than from the interval $[1,1]$ to $[-1,1]$. And this property is of course inverted for $b_{2}$.

A comparison of the fused bba $m_{1} \oplus m_{2}$ with the initial bbas $m_{1}$ and $m_{2}$ makes apparent a global forward move of the density. This just means that the fused bba is put on intervals with less imprecision, and possibly on some intervals with negative width (ie. associated with a degree of contradiction). Of course there is nothing surprising here, since information fusion will reduce imprecision and produce some contradiction! It is also noticed that the fused bba is centered around the interval $[0,0]$. This result matches perfectly the fact that $m_{1}$ and $m_{2}$, and their related sensors, put more belief respectively over the interval $[-1,0]$ and the interval $[0,1]$; and of course $[-1,0] \cap[0,1]=[0,0]$.

\section{Conclusion}

A problem of continuous information fusion has been investigated and solved in the DSmT paradigm. The conceived method is versatile and is able to specify the typical various degrees of contradiction of a DSm model. It has been implemented efficiently for a bounded continuous information. The work is still prospective, but applications should be done in the future on localization problems. At this time, the concept is restricted to one-dimension informations. However, works are now accomplished in order to extend the method to multiple-dimensions domains. 


\section{pre-bba $m m_{2}$}

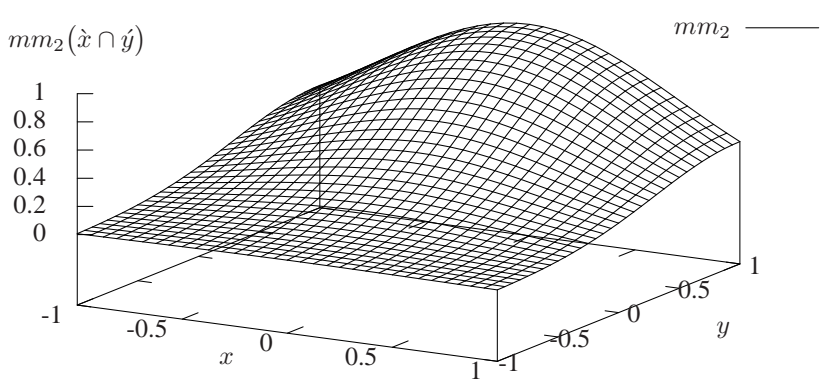

Figure 2: Non normalized bba $m m_{2}$

\section{bba $m_{1}$}

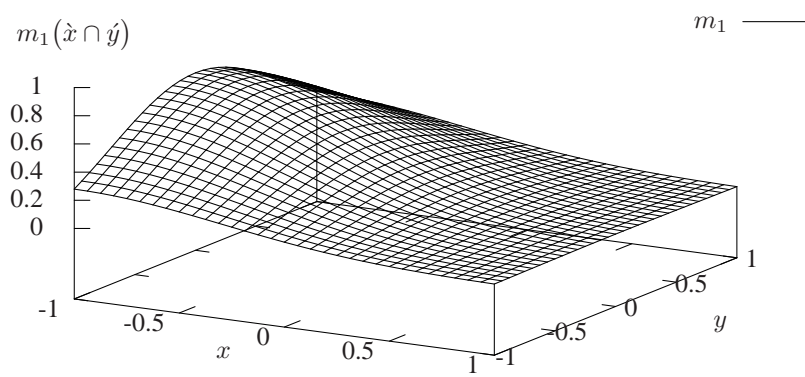

Figure 3: Basic belief assignment $m_{1}$ 


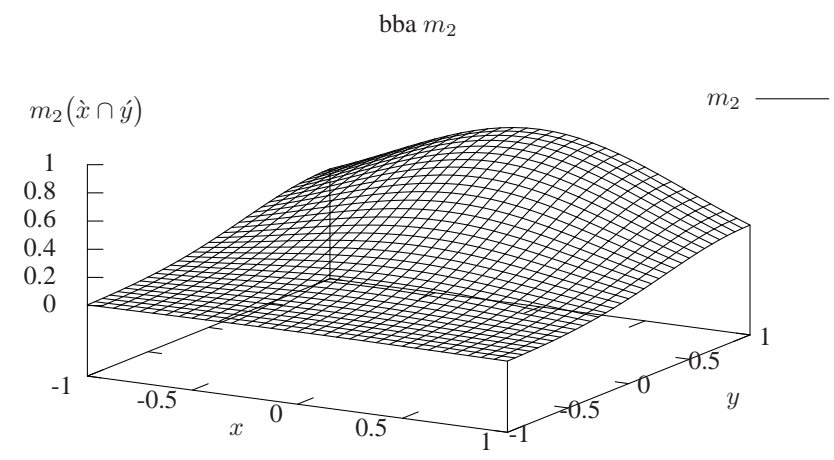

Figure 4: Basic belief assignment $m_{2}$

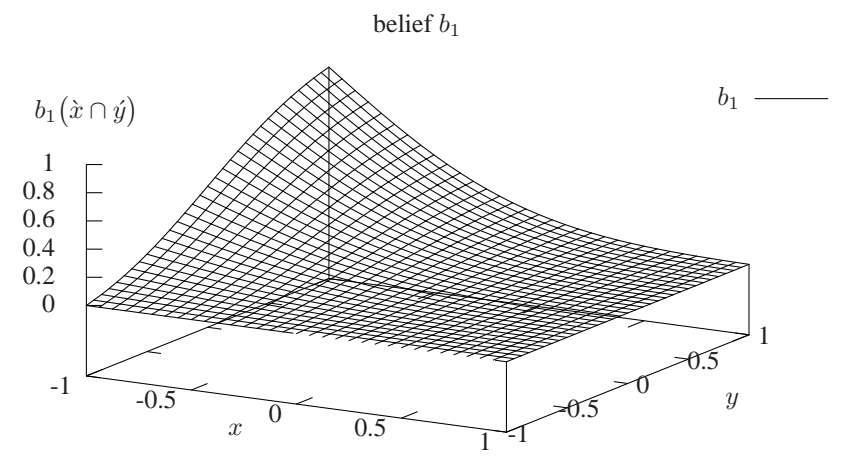

Figure 5: Belief function $b_{1}$ 


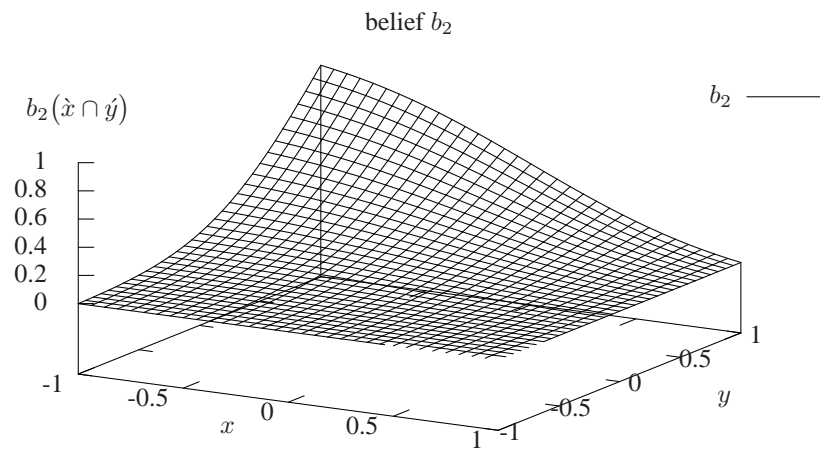

Figure 6: Belief function $b_{2}$

\section{bba $m_{1} \oplus m_{2}$}

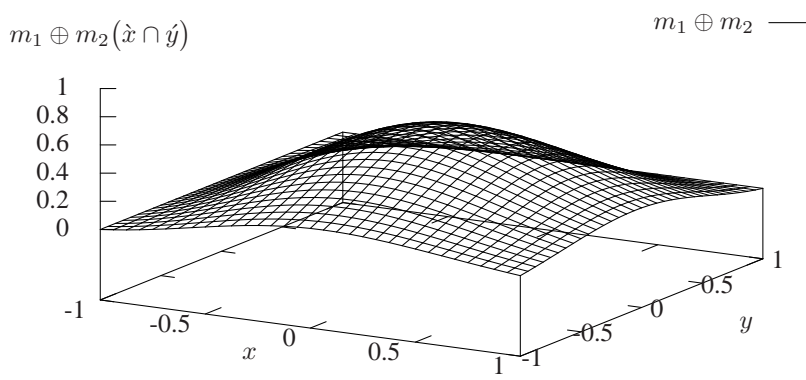

Figure 7: Fused bba $m_{1} \oplus m_{2}$ 


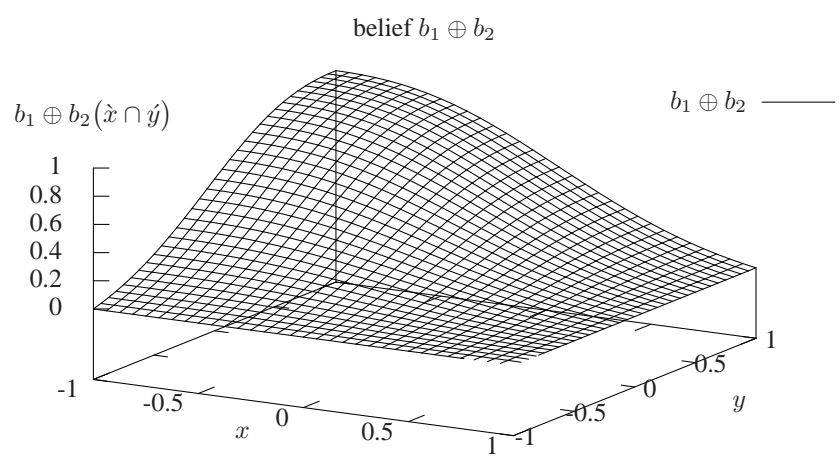

Figure 8: Fused bba $b_{1} \oplus b_{2}$

\section{References}

[1] F. Smarandache and J. Dezert editors, Advances and Applications of DSmT for Information Fusion, American Research Press, Rohoboth, 2004.

www. gallup. unm. edu/ smarandache/DSmT.htm

[2] A. Tchamova, J. Dezert, Tz. Semerdjiev, P. Konstanttinova, Target Tracking with Generalized Data Association based on the General DSm Rule of Combination, Conference Fusion 2004, Sweden, 2004.

[3] S. Corgne, L. Hubert-Moy, G. Mercier, J. Dezert, Application of DSmT for Land Cover Change Prediction, in Advances and Applications of DSmT for Information Fusion, American Research Press, Rohoboth, 2004.

[4] P. Smets and R. Kennes, The transferable belief model, Artificial Intelligence 66, pp 191-234, 1994

[5] B. Ristic and P. Smets, Belief function theory on the continuous space with an application to model based classification, IPMU 2004, Italy, 2004.

[6] T. M. Strat, Continuous belief functions for evidential reasoning, AAAI 1984, pp 308-313.

[7] A. P. Dempster, Upper and lower probabilities induced by a multiple valued mapping, Ann. Math. Statistics, no. 38, pp. 325-339, 1967.

[8] Shafer G., A Mathematical Theory of Evidence, Princeton Univ. Press, Princeton, NJ, 1976. 
FRÉDÉRIC DAMBREVILLE Frederic Dambreville studied mathematics, logic, signal and image processing. He received the Ph.D. degree in signal processing and optimization, from the university of Rennes, France, in 2001. He enjoyed a stay in California (U.S.A.) and worked as a postdoctorate in the Naval Postgraduate School at Monterey in 2001/2002. In 2002, he joined the department image, perception and robotic of the CTA laboratory (Delegation Generale pour l'Armement), France. His main interests are in optimization, optimal planning, game theory, simulation methods, data\&sensor fusion, Markov models\&Bayesian networks, Logic\&Conditional logic. His most recent works are about rare event simulation (e.g. Cross-Entropy optimization), optimal decision with partial observation, hierachical system optimization, Scheduling, modal\&Bayesian logic, and DSmT.

Délégation Générale pour l'Armement, DGA/CEP/GIP/SRO

16 Bis, Avenue Prieur de la Côte d'Or

Arcueil, F 94114, France

Web: http://www.FredericDambreville.com

Email: http://email.FredericDambreville.com 